\title{
Inhibitory effects of pu-erh tea on alpha glucosidase and alpha amylase: a systemic review
}

\author{
Chiung-Ying Yang ${ }^{1}$, Yea-Yin Yen², Kuang-Chen Hung ${ }^{3,4,5}$, Shang-Wei Hsu', Shou-Jen Lan ${ }^{1,6}$ and Hsin-Cheng Lin $\mathbb{1}^{1,4,5}$
}

\begin{abstract}
Objective: Pu-erh tea was presumed to have anti-hyperglycemic effects via inhibition on alpha-amylase and alphaglucosidase. However, no integerated literatures were published to substantiate such presumption.

Methods: Current study adopted systemic review method to validate inhibitory effects on alpha amylase and alphaglucosidase. Five English databases (PubMed, EBSCO, SCOPUS, Cochrane Library, Web of Science) and three Chinese ones (Airti Library, CNKI Library, and Google Scholar) were searched up to 22 March 2018 for eligible literatures, using keywords of Pu-erh, Pu'er, alpha-amylase or alpha-glucosidase.
\end{abstract}

Results: Six studies exploring inhibitory effects on alpha-glucosidase and seven on alpha-amylase were included for systemic review. Though results showed pu-erh tea has significant inhibitory effects on alpha-amylase and alphaglucosidase, high heterogeneity was detected among studies included.

Conclusions: High heterogeneity may be due to complex alterations of chemicals under different degrees of fermentation. More future studies are required to further identify principal bioactive component(s) at work.

\section{Introduction}

Tea drinking has been part of Chinese culture for centuries. Tea was widely regarded to have many health benefits. Green tea, black tea, or oolong teas were all good for rats on high fructose diet. Pu-erh tea is made from camellia sinensis plant's leaves and stems, also the same principal ingredient for green, oolong, and black teas. Different teas were processed and manufactured using distinct preparation procedures, where green tea is not fermented, oolong partially fermented, black tea fully fermented, and pu-erh post-fermented. Pu-erh tea has long been espoused as a health beverage in oriental societies including China and Taiwan. China's Compendium of Materia Medica contains passages of: "Pu-erh tea

\footnotetext{
Correspondence: Hsin-Cheng Lin (p840025@gmail.com)

1 Department of Healthcare Administration, Asia University, Taiwan, Taiwan

${ }^{2}$ Department of Oral Hygiene, College of Dental Medicine, Kaohsiung Medical University, Taichung, Taiwan

Full list of author information is available at the end of the article.
}

taste bitter and promote fat metabolism. Moreover the $\mathrm{Pu}$-erh tea paste looks like black paint, it can sober up the drunken people."

$\mathrm{Pu}$-erh tea is primarily grown in Yunnan Province of China. Pu-erh tea extracts were found to significantly improve plasma concentrations of glucose, insulin, triglycerides, and free fatty acids ${ }^{1-3}$. Recent studies have further confirmed that catechins, caffeine, polyphenols, amino acids, and polysaccharides in pu-erh tea extracts also have beneficial effects on maintaining in vivo glucose homeostasis in type 2 diabetes mice ${ }^{4}$. Pu-erh tea's distinctive effects could potentially be attributed to a series of complex changes in chemicals during post-fermentation stage. These complex chemical changes in turn were found to lead to pu-erh's great health benefits, such as lower atherosclerotic risk ${ }^{5}$, weight reduction ${ }^{6}$, and antihyperglycaemic effect, especially ${ }^{7}$.

Starch is the most important source of carbohydrates for human and several different animal species while $\alpha$ - 
amylase and $\alpha$-glucosidase are two primary enzymes involved in carbohydrate digestion. Salivary and pancreatic $\alpha$-amylases cleave the $\alpha-(1 \rightarrow 4)$-D-glycosidic bonds of starch at random sites, forming smaller oligosaccharides or disaccharides. Alfa glucosidase is located in brush borders of small intestines, where this digestive enzyme hydrolyzes terminal non-reducing $\alpha-1 \rightarrow 4$ linkage of oligosaccharides or disaccharides and releases glucose molecules (Fig. 1).

Alpha glucosidase plays an important role in regulating postprandial blood glucose levels in human body ${ }^{8}$ and its inhibitors, capable of suppressing postprandial hyperglycaemia, are usually used to prevent or treat type II diabetes ${ }^{9}$. Alpha amylase is basically a calcium metalloenzyme, acting as a catalyst in reactions involving alpha-1,4 glycosidic linkages hydrolyses of starch, amylopectin, amylose, glycogen, and numerous maltodextrins, and is therefore responsible for starch digestion. In order to make use of starch, the human digestive system, with the help of the enzyme amylases, break down the polymer to smaller assimilable sugars, which is eventually converted to the individual basic glucose units. Under some circumstances, such as excess activity of amylase enzymes, insulin deficiency, or resistance to insulin, blood glucose level may rise and in turn result in hyperglycaemia.

Carbohydrates from pu-erh tea polysaccharides (PTPS) can inhibit both alpha glucosidase and alpha amylase ${ }^{10}$. To offer alternative or even better solutions for diabetes patients to control their blood sugar level, this study aims to explore pu-erh tea's effects on diabetes mellitus. Adopting systemic literature review method and analyzing all eligible research articles included for analysis, this study investigated pu-erh tea's inhibitory effects on alphaglucosidase and alpha-amylase in lowering blood glucose and made comprehensive conclusions regarding pu-erh tea's effectiveness on regulating blood sugar level.

\section{Method}

Systemic literature review method was used to determine inhibitory effects of pu-erh tea on alpha-glucosidase and alpha-amylase.

\section{Information sources and searches}

Eight bibliographic databases were searched: (1) PubMed, (2) EBSCO, (3) SCOPUS, (4) Cochrane Library, (5) Web of science, (6) Airiti Library, (7) China National Knowledge Infrastructure (CNKI), and (8) Google Scholar. Among the eight databases, Airiti Library and China National Knowledge Infrastructure (CNKI) were searched for literatures in Chinese. Furthermore, Google Scholar was also searched for eligible studies either in English or Chinese. The Search terms are listed in Table 1.

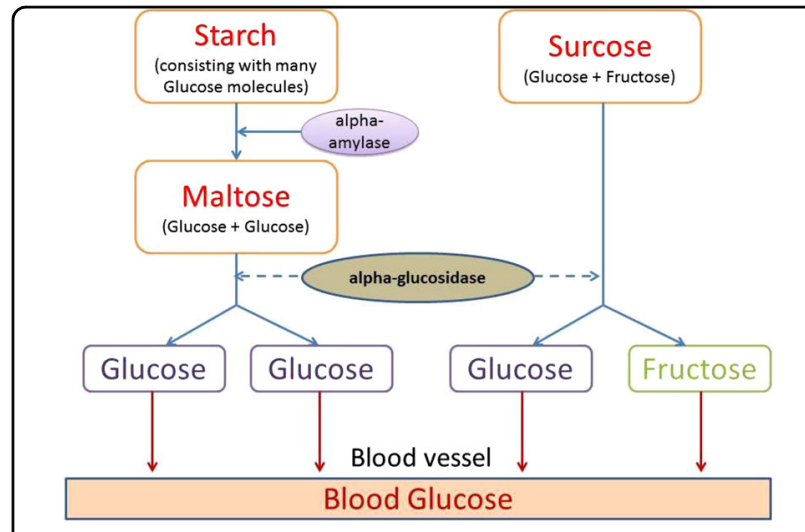

Fig. 1 Hydrolysis of starch to glucose as catalyzed by alpha-amylase and alpha-glucosidase

Table 1 Search terms

\begin{tabular}{|c|c|c|}
\hline Search Strings & AND & Search strings \\
\hline Pu-erh & & Alpha glucosidase \\
\hline OR & & OR \\
\hline Pu'er & & Alpha amylase \\
\hline
\end{tabular}

\section{Study selection}

A total of 71 articles were initially retrieved from the eight databased searched (five English databases, two Chinese databases, and Google Scholar searching for articles either in English or Chinese).

Out of the 71 articles retrieved, 45 of them were retained after removing 16 duplicate or review articles. Current study authors further excluded 32 articles deemed unsuitable for systemic review by examining their respective title and abstract.

The remaining 13 articles were further reduced to 12 , after excluding 0 studies determined irrelevant to the purpose of the current study and one non full-text articles.

For the remaining 12 full-text articles selected after abovementioned screenings, all of them were unanimously determined by all research authors to pass PICO (population, interventions, compare, and outcome) criteria and were in turn included for full review.

It is noteworthy that 1 study Du, Peng ${ }^{4}$ included for full review was determined to relate to inhibition effects on both alpha amylase and alpha glucosidase, consequently making the final full-review articles count to 13 from 12 .

Also noteworthy is that a trial was considered eligible and in turn included for full review if it addressed any puerh tea intervention effects, whether in vivo or in vitro. The outcome column in Table 2 highlighted inhibition 
Table 2 System review results of Pu-erh tea inhibition effects on alpha glucosidase

\begin{tabular}{|c|c|c|}
\hline Study & Experiment subjects & outcome \\
\hline Li et al. ${ }^{11}$ & Alpha glucosidase inhibition assay & $\begin{array}{l}\text { 1. (-)-epigallocatechingallate (EGCG) and (-)-epicatechingallate (ECG), as potent } \\
\text { alpha glucosidase inhibitors, were found in pu-erh tea. } \\
\text { 2. The } \mathrm{IC}_{50} \text { values of EGCG and ECG on alpha-glucosidase were } 175.1 \text { and } \\
246.9 \mu \mathrm{M} \text {, respectively, and both were lower than that of acarbose } \\
\left(\mathrm{IC}_{50}=3553.0 \mu \mathrm{M}\right) \text {, a commercial alpha glucosidase inhibitor. } \\
\text { 3. Different concentrations have different Inhibition rates }(1.017 .1+-1.3) \text {. }\end{array}$ \\
\hline Yang et al. ${ }^{12}$ & Alpha glucosidase inhibition assay & $\begin{array}{l}\text { In the concentration } 2.5 \mathrm{mg} / \mathrm{ml} \text {, the inhibition rate of acarbose: } 60.16 \% \text {, ethyl } \\
\text { acetate fraction: } 82.52 \% \text {, n-butanol fraction: } 65.84 \% \text {, water fraction: } 58.22 \% \text {, water } \\
\text { extract: } 52.29 \% \text {. }\end{array}$ \\
\hline Du et al. $^{4}$ & $\begin{array}{l}\text { Alpha glucosidase inhibition assays for the WEPT } \\
\text { were conducted using rat small intestinal sucrose and } \\
\text { maltase in vitro }\end{array}$ & $\begin{array}{l}\text { WEPT showed inhibitory effects on rat intestinal sucrose, maltase, and porcine } \\
\text { pancreatic amylase, as shown in Table } 3 \text {, but are less potent compared to } \\
\text { acarbose }\left(\mathrm{IC}_{50}=4.35 \pm 0.59,6.63 \pm 0.70 \text {, and } 64.19 \pm 6.77 \mu \mathrm{mol} / \mathrm{L} \text { for sucrose, }\right. \\
\text { maltase, and amylase, respectively). }\end{array}$ \\
\hline Huang et al. ${ }^{13}$ & Alpha glucosidase inhibition assay & $\begin{array}{l}\text { 1. EP exhibited the strongest inhibitory potential, which increased from } 25.96 \% \text { to } \\
87.2 \% \text { when the concentration increased from } 4 \mu \mathrm{g} / \mathrm{ml} \text { to } 125 \mu \mathrm{g} / \mathrm{ml} \text {. } \\
\text { 2. EF, the inhibitory effect increased from } 20.43 \% \text { to } 84.77 \% \text { at the same range of } \\
\text { concentrations. } \\
\text { 3. Meanwhile, } \mathrm{AE}, \mathrm{R} \text { and } \mathrm{SF} \text { also showed a great inhibition on a-glycosidase with } \\
\text { an inhibitory effect of } 78.85 \%, 69.94 \% \text { and } 69.47 \% \text { at a concentration of } 125 \mathrm{ug} / \mathrm{ml} \text {, } \\
\text { which were higher than that of acarbose at the same concentration }(40.84 \%) \text {. }\end{array}$ \\
\hline Deng et al. ${ }^{10}$ & Enzyme assay of a-glucosidase activity & $\begin{array}{l}\text { 1. The IC50 of acarbose and the IC50s of TPSs from Feng Huang Dan Cong, Da } \\
\text { Hong Pao and pu-erh tea (1-year old, } 3 \text {-year old and 5-year old) on alpha } \\
\text { glucosidase activity were } 207.195,5.653,2.286,2.192,0.583 \text {, and } 0.438 \mu \mathrm{g} / \mathrm{ml} \text {. } \\
\text { 2. PTPS had more significant effects on a-glucosidase than acarbose did. }\end{array}$ \\
\hline Wang et al. ${ }^{14}$ & Alpha glucosidase inhibition assay & $\begin{array}{l}\text { 1. The results showed that EtOAc fraction had a moderate inhibitory activity with } \\
\mathrm{IC}_{50} \text { values of } 14.4 \mu \mathrm{g} / \mathrm{mL} \text { against sucrose and } 11.4 \mu \mathrm{g} / \mathrm{mL} \text { against maltase, } \\
\text { respectively. } \\
2 \text {. The inhibitory effects of compounds } 1-17 \text { were also measured using the same } \\
\text { methods. (-)-Epigallo-catechin-3-O-gallate (15) showed a moderate activity with } \\
\mathrm{IC}_{50} \text { values of } 32.5 \mu \mathrm{mol} / \mathrm{L} \text { against sucrose and } 1.3 \mu \mathrm{mol} / \mathrm{L} \text { against maltase, } \\
\text { respectively. }\end{array}$ \\
\hline
\end{tabular}

rate of alpha-amylase or alpha-glucosidase of individual studies included for full review. Below excluding criteria were employed to remove ineligible studies: review article, duplicate article, non-full-text article, study without data on blood glucose change, and non-animal trial.

\section{Results}

\section{Search results}

In the first stage of the literature search, a total of 71 studies were identified. Among the 71 potentially eligible articles initially searched, 21 articles were published in English (2 from PubMed, 2 from EBSCO, 5 from SCOPUS, 0 from Cochrane, 1 from Web of Science, and 11 from Google Scholar) and 50 studied were published in Chinese. (7 from Airti Library, 9 from China National Knowledge Infrastructure (CNKI), and 34 from Google Scholar.

The second stage was employed to further examine searched articles and determine their respective eligibility, per inclusion and exclusion criteria jointly agreed by all research authors, with duplicate or ineligible articles removed from being included in final review accordingly.

After the aforementioned search strategy and two rounds of eligibility screening process, a total of 12 articles were selected and included in the current study's systemic review: six studies exploring pu-erh tea's inhibitory effects on alpha-glucosidase and another seven studies regarding its inhibitory effects on $\alpha$-amylase (Fig. 2).

\section{Inhibitory effects on a-glucosidase}

Li et al. ${ }^{11}$ examined inhibitory effects on alfa glucosidase of EGCG and ECG, two compounds found in pu-erh tea and their activities expressed in dose-dependent manner as opposed to those of acarbose, a commercial alphaglucosidase inhibitor anti-diabetic drug used to treat type 2 diabetes. $\mathrm{IC}_{50}$ values of EGCG and ECG were determined to be 175.1 and $246.9 \mu \mathrm{M}$ respectively, much lower than that of acarbose $(3553.0 \mu \mathrm{M})$. Kinetic studies further revealed that both EGCG and ECG inhibit alphaglucosidase in a non-competitive manner.

Yang et al. ${ }^{12}$ researched water extract of pu-erh tea (WEPT) and its fractions extracted by chloroform, ethyl acetate, n-butanol, and water. Research results showed that pu-erh's fractions had different inhibitory effects on alpha-glucosidase activity when their concentration was $2.5 \mathrm{mg} / \mathrm{ml}$. Positive controls of their study: acarbose, ethyl acetate fraction, n-butanol fraction, water fraction, and water extract revealed a noticeable difference in inhibitory activity, with inhibition rates of $60.16 \%, 82.52 \%, 65.84 \%$, $58.22 \%$, and $52.29 \%$ respectively. Varying effects of water extract and its different extraction fractions from pu-erh 


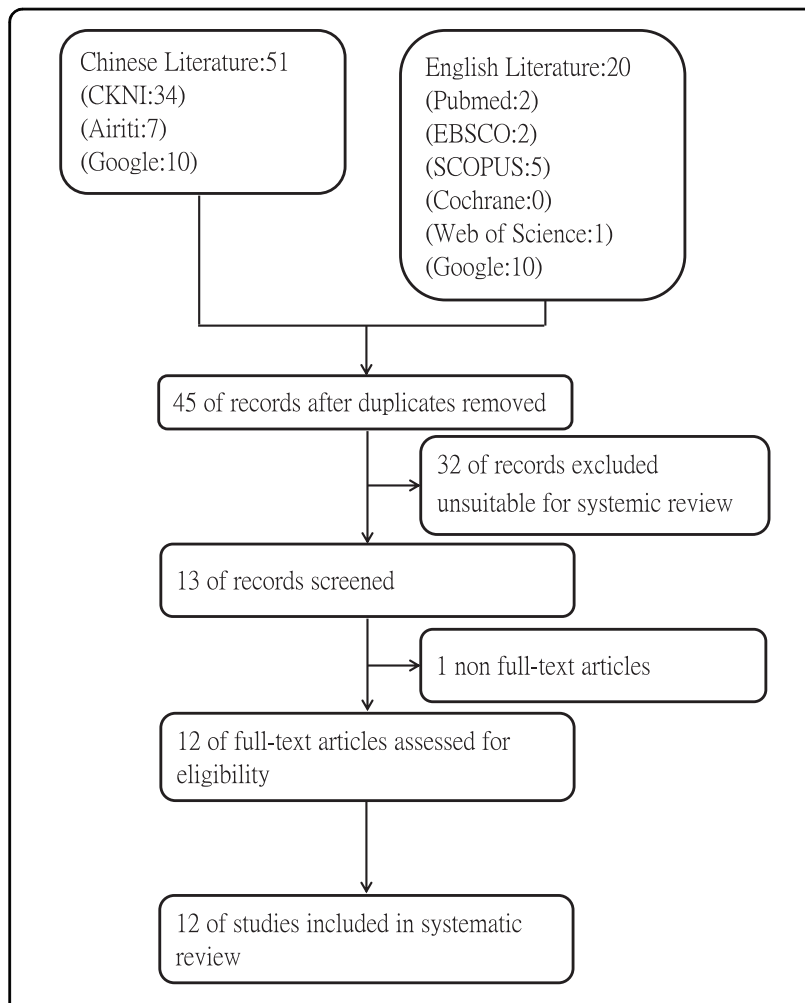

Fig. 2 Flow chart describing literature extraction process

tea on alpha glucosidase activity were found to be dictated by difference in chemical composition.

$\mathrm{Du}$ et al. ${ }^{4}$ used high-performance liquid chromatography and colorimetric methods to analyze tea catechins, caffeine, polyphenols, amino acids, and polysaccharides of WEPT. In this study, $\alpha$-glucosidase inhibition assays for WEPT were conducted, using rat small intestinal sucrase and maltase in vitro. In their study, WEPT was found to have inhibitory effects on rat intestinal sucrase, maltase, and porcine pancreatic amylase (as shown in Table 3), but WEPT's inhibitory effects were found to be less potent than those of acarbose. $\left(\mathrm{IC}_{50}=4.35 \pm 0.59,6.63 \pm 0.70\right.$, and $64.19 \pm 6.77 \mu \mathrm{mol} / \mathrm{L}$ for sucrase, maltase, and amylase respectively) The results showed that WEPT dosedependently and significantly increased glucose uptake by HepG2 cells and inhibited rat intestinal sucrase, maltase, and porcine pancreatic amylase activity.

Huang et al. ${ }^{13}$ established a procedure to obtain puerh's aqueous extract and its fraction enriched with active constituents. The authors also used this procedure to evaluate bioactivities of pu-erh's aqueous extract and its fractions, including antioxidant activity and inhibitory potential against alpha-glycosidase in vitro as well as effect on postprandial hyperglycemia in alloxan-induced diabetic mice. Their study also found that tea polyphenols, tea polysaccharides, caffeine, protein, amino acids were accumulated in several fractions after solvent extraction despite not being separated completely. Furthermore, 95\% ethanol precipitate and ethyl acetate fraction possessed remarkable antioxidant activities and potent inhibitory effects on alpha glycosidase in vitro. The authors also observed that all extracts showed a concentration-dependent inhibition on a-glycosidase. Study results further showed that EP exhibited the strongest inhibitory potential, increasing from 25.96 to $87.2 \%$ with concentration level increasing from $4 \mu \mathrm{g} / \mathrm{ml}$ to $125 \mu \mathrm{g} / \mathrm{ml}$. EF was found to rank second in terms of inhibitory effect which increased from 20.43 to $84.77 \%$ at the same range of concentrations. The authors further found that $\mathrm{AE}, \mathrm{R}$, and $\mathrm{SF}$ also showed a great inhibition on a-glycosidase with an inhibitory effect of $78.85,69.94$, and $64.97 \%$ at a concentration of $125 \mu \mathrm{g} / \mathrm{ml}$, much higher than that of acarbose at the same concentration (40.84\%). Results derived from assay performed also showed that CF exhibited the weakest inhibitory potential against a-glycosidase, with highest inhibition of $36.94 \%$ at a concentration of $125 \mu \mathrm{g} / \mathrm{ml}$.

Deng et al. ${ }^{10}$ detected a group of carbohydrates found in PTPS that can inhibit alpha-glucosidase. When comparing inhibition effects of PTPS and acarbose on alpha glucosidase, they found that PTPS from pu-erh tea (1-year old, 3-year old, and 5-year old) significantly inhibited alpha glucosidase activity but did not affect alpha amylase activity. In comparing inhibitory effects on alpha glucosidase activity of acarbose and PTPS found in different types of teas, their study have identified their respective $\mathrm{IC}_{50}$ values to be: (1) $207.195 \mu \mathrm{g} / \mathrm{ml}$ for acarbose, (2) $5.653 \mu \mathrm{g} / \mathrm{ml}$ for Feng Huang Dan Cong (special types of oolong from Feng Huang Shan or "Phoenix Mountains" in Guandong province, China.), (3) $2.286 \mu \mathrm{g} / \mathrm{ml}$ for Da Hong Pao (a heavily oxidized, dark oolong tea), (4) $2.192 \mu \mathrm{g} / \mathrm{ml}$ for 1-year old pu-erh tea, (5) $0.583 \mu \mathrm{g} / \mathrm{ml}$ for 3-year old pu-erh tea, and (6) $0.438 \mu \mathrm{g} / \mathrm{ml}$ for 5-year old pu-erh tea. Results from their study have clearly indicated that PTPS had more significant effects on alpha glucosidase than did acarbose.

Wang et al. ${ }^{14}$ found that WEPT showed potential in vitro hypoglycemic effects. The results showed that EtOAc fraction had moderate inhibitory activity with $\mathrm{IC}_{50}$ values of $14.4 \mu \mathrm{g} / \mathrm{mL}$ against sucrose and $11.4 \mu \mathrm{g} / \mathrm{mL}$ against maltase (Acarbose $\mathrm{IC}_{50}$ values of $0.262 \mu \mathrm{g} / \mathrm{mL}$ against sucrose and $0.084 \mu \mathrm{g} / \mathrm{mL}$ against maltase).

\section{Inhibitory effect on a-amylase}

Zhang et al. ${ }^{15}$ established that alpha amylase inhibition of raw pu-erh tea increased with the extension of fermentation time. The inhibition rate dropped, and reached the lowest in mid stage fermentation, while in late fermentation, the inhibition increased and $\alpha$-amylase inhibition of product tea was stronger, with inhibition rates ranging from 45.61 to $12.44 \%$. 
Table 3 System review results of pu-erh's inhibition effects on alpha amylase

\begin{tabular}{|c|c|c|}
\hline Study & Experiment subjects & Outcome \\
\hline Zhang et al. ${ }^{15}$ & Alpha amylase inhibition assay & $\begin{array}{l}\text { Alfa-amylase inhibition of raw materials increased with the extension of } \\
\text { fermentation time, the inhibition rate dropped, and reached the lowest in mid } \\
\text { stage fermentation, while in late fermentation, inhibition increased and aamylase } \\
\text { inhibition of product tea was stronger. }\end{array}$ \\
\hline Zhang et al. ${ }^{16}$ & Alpha amylase inhibition assay & $\begin{array}{l}\text { The results showed that the best inhibition rate is } 32.68 \% \text { in water temperature of } \\
90^{\circ} \mathrm{C} \text {, pu-erh tea/water ratio of } 1: 20 \text {, and extraction time lasting } 1 \mathrm{~h} \text {. }\end{array}$ \\
\hline Zhang et al. ${ }^{17}$ & Alpha amylase inhibition assay & $\begin{array}{l}\text { The result showed that Gallic acid had a strong inhibition on alpha amylase, with } \\
\text { inhibition rate of } 63.76 \% \text {. }\end{array}$ \\
\hline Du et $\mathrm{al}^{4}{ }^{4}$ & $\begin{array}{l}\text { Alpha glucosidase inhibition assays for the WEPT were } \\
\text { conducted using rat small intestinal sucrose and maltase } \\
\text { in vitro }\end{array}$ & $\begin{array}{l}\text { WEPT showed inhibitory effects on rat intestinal sucrose, maltase, and porcine } \\
\text { pancreatic amylase, as shown in Table } 3 \text {, but are less potent compared to } \\
\text { acarbose }\left(I C_{50}=4.35 \pm 0.59,6.63 \pm 0.70 \text {, and } 64.19 \pm 6.77 \mu \mathrm{mol} / \mathrm{L} \text { for sucrose, }\right. \\
\text { maltase, and amylase, respectively). }\end{array}$ \\
\hline Zhou et $\mathrm{al}^{18}$ & Alpha amylase inhibition assay & $\begin{array}{l}\text { 1. Prolonged storage years will increase inhibition effect on alpha amylase activity. } \\
\text { 2. The inhibition rate ranges from } 10 \text { to } 22 \% \text {. The regression model: } y \\
\text { (alphaamylase inhibition rate) }=-31.43+0.748 x ; x \text { : theabrownin, TB; } R^{2}=97.4 \% \text {. }\end{array}$ \\
\hline $\begin{array}{l}\text { Liu and } \\
\text { Huang }\end{array}$ & Alpha amylase inhibition assay & $\begin{array}{l}\text { 1. Pu-erh has inhibition effects on alpha amylase. } \\
\text { 2. Regression equation: } y=0.0008 \times 3+0.0075 \times 2-0.0106 x+0.1236, x \text { : extracted } \\
\text { tea concentration; } R^{2}=0.998 \text {. }\end{array}$ \\
\hline Wu et al. ${ }^{20}$ & Alpha amylase inhibition assay & $\begin{array}{l}\text { Optimal temperature of alpha amylase inhibitor acting is } 70^{\circ} \mathrm{C} \text {, and suppression } \\
\text { ratio of alpha Amylase inhibitor in these teas is } \| \text { ex }>\text { aged pu-erh tea }>\text { extra- } \\
\text { strong tea }>\text { dragon well tea }>\text { fresh pu-erh tea. }\end{array}$ \\
\hline
\end{tabular}

Zhang et al. ${ }^{16}$ examined the relationship between water temperature, pu-erh tea/water ratio, extraction time, and amylase inhibition rate. The results showed that the best inhibition rate is $32.68 \%$, with water temperature of $90^{\circ} \mathrm{C}$, pu-erh tea/water ratio being 1:20, and extraction time lasting $1 \mathrm{~h}$ respectively.

Zhang et al. ${ }^{17}$ explored chemical constituents of Pu-erh tea and its inhibition effect on $\alpha$-amylase. Uracil and gallic acids were isolated and identified respectively from pu-erh tea. The result showed that gallic acid had a strong inhibition on alpha amylase, with inhibition rate of $63.76 \%$.

Zhou et al. ${ }^{18}$ looked into quality change of pu-erh tea, using a spectrophotometer to evaluate inhibition effects on a-amylase of pu-erh teas with different storage years. Results indicated that with storage year prolonging, its inhibition effect on alpha amylase activity also increased, with inhibition rate ranging from 10 to $22 \%$. The regression model: $y$ (alpha-amylase inhibition rate) $=-31.43+$ $0.748 x$ (x: theabrownin or TB); $R^{2}$ is $97.4 \%$.

Liu et al. ${ }^{19}$ concluded that pu-erh has an inhibition effect on alpha amylase, with its regression equation shown as: $y=0.0008 \mathrm{x}^{3+} 0.0075 \mathrm{x}^{2}-0.010 \mathrm{x}+0.1236(\mathrm{X}$ : extracted tea concentration); $R^{2}=0.998$.

Wu et al. ${ }^{20}$ based their study on alpha-amylase inhibitor in several teas. Their study found below results: (1) Optimal temperature of alpha amylase inhibitor acting is $70{ }^{\circ} \mathrm{C}$, (2) Suppression ratio of alpha Amylase inhibitor in these teas is Ilex $>$ aged pu-erh tea $>$ extra-strong tea $>$ dragon well tea $>$ fresh pu-erh tea.

$\mathrm{Du}$ et al. ${ }^{4}$ used high-performance liquid chromatography and colorimetric methods to analyze tea catechins, caffeine, polyphenols, amino acids, and polysaccharides of
WEPT. Results of their research showed that WEPT dosedependently and significantly inhibited pancreatic amylase activities in both mice and porcine.

\section{Discussion \\ Inhibitory effects on a-glucosidase}

Lin et al. ${ }^{7}$ used systemic review and meta-analysis in their study and found that $\mathrm{Pu}$-erh tea can help regulate and maintain adequate level of blood sugar. However, the specific bioactive ingredient(s) or mechanism for lowering hyperglycaemia was still unknown for certainty. According to the authors' research findings, pu-erh tea's antihyperglycaemia mechanism was associated with the inhibition either on $\alpha$-glucosidase or $\alpha$-amylase.

But Pu-erh tea's inhibitory effects on $\alpha$-glucosidase as compared to those of Acarbose remained uncertain. Li et al. ${ }^{11}$ and Deng et al. ${ }^{10}$ indicated that pu-erh teas were potent alpha glucosidase inhibitors, with their $\mathrm{IC}_{50}$ values much lower than those of acarbose. However, Wang et al. ${ }^{14}$ found that WEPT had only moderate inhibitory activity with IC50 values of $14.4 \mu \mathrm{g} / \mathrm{mL}$ against sucrose and $11.4 \mu \mathrm{g} / \mathrm{mL}$ against maltase (Acarbose IC50 values of $0.262 \mu \mathrm{g} / \mathrm{mL}$ against sucrose and $0.084 \mu \mathrm{g} / \mathrm{mL}$ against maltase).

\section{Inhibitory effects on a-amylase}

Zhang et al. ${ }^{17}$ found that gallic acid had a strong inhibition effect on alpha amylase, with inhibition rate of $63.76 \%$. Findings derived from the authors' review showed that the inhibition effect was primarily associated with fermentation time, water temperature, pu-erh tea/water ratio, among others. 


\section{Conclusion}

With its rapidly increasing prevalence, chronic disease course, and gravely debilitating clinical complications, Diabetes mellitus has become one of the primary threats to human health. As such, postprandial hyperglycemia control has naturally become an urgent issue in prevention, treatment, and control of diabetes.

Alpha-glucosidases are glycoside hydrolases found on luminal surface of enterocytes containing maltase/glucoamylase and sucrase/isomaltase activities. Alphaglucosidase inhibitors also favorably affected several cardiovascular activities. The risk factors, such as obesity, hypertension, and high glycaemic variability was at little to no risk for hypoglycemia ${ }^{21}$.

More and more studies have been conducted to examine inhibition effects on alpha glucosidase and alpha amylase of pu-erh tea, aimed at exploring physiological and functional results for the prevention and treatment of type 2 diabetes. It is noteworthy that high heterogeneity was detected among these studies. Other studies also indicated that health-promoting benefits of tea drinking could be the summation of effects by all individual ingredients in pu-erh, especially polyphenols. One possible reason is that complex alterations may occur in chemicals under different degrees of fermentation, resulting in an obscure understanding of chemical compositions in pu-erh teas, unlike the much studied and more thoroughly understood green (unfermented) teas. As such, more future studies are required to further identify principal bioactive component(s) at work and their actual mechanisms.

\section{Author details \\ ${ }^{1}$ Department of Healthcare Administration, Asia University, Taiwan, Taiwan. ${ }^{2}$ Department of Oral Hygiene, College of Dental Medicine, Kaohsiung Medical University, Taichung, Taiwan. ${ }^{3}$ Central Taiwan University of Science and Technology, Taichung, Taiwan. ${ }^{4}$ Taichung Armed Forces General Hospital, Taichung, Taiwan. ${ }^{5}$ National Defense Medical Center, Taipei, Taiwan. ${ }^{6}$ Department of Medical Research, China Medical University Hospital, China Medical University, Taichung, Taiwan}

\section{Conflict of interest}

The authors declare that they have no conflict of interest.

\section{Ethical approval}

This review article did not require informed consent or approval by the ethics committee.

\section{Publisher's note}

Springer Nature remains neutral with regard to jurisdictional claims in published maps and institutional affiliations.

Received: 11 June 2019 Revised: 21 July 2019 Accepted: 25 July 2019

Published online: 27 August 2019

\section{References}

1. Yang, M., Wang, C. \& Chen, H. Green, oolong and black tea extracts modulate lipid metabolism in hyperlipidemia rats fed high-sucrose diet. J. Nutr. Biochem. 12, 14-20 (2001)

2. Wu, L. Y. et al. Green tea supplementation ameliorates insulin resistance and increases glucose transporter IV content in a fructose-fed rat model. Eur. J. Nutr. 43, 116-124 (2004).

3. Hsu, S. H. Effect of Pu-erh tea on blood sugar and lipid in STZ-induced diabetic rats Department of Food and Nutrition.(Providence University, Taichung, Taiwan, 2009).

4. Du, W.-H. et al. Hypoglycemic effect of the water extract of Pu-erh tea. J. Agric. Food Chem. 60, 10126-10132 (2012).

5. Hou, Y. et al. Pu-erh tea aqueous extracts lower atherosclerotic risk factors in a rat hyperlipidemia model. Exp. Gerontol. 44, 434-439 (2009).

6. Oi, Y., Hou, I. C., Fujita, H. \& Yazawa, K. Antiobesity effects of Chinese black tea (Pu-erh tea) extract and gallic acid. Phytother. Res. 26, 475-481 (2012).

7. Lin, H.-C. et al. Systematic review and meta-analysis of anti-hyperglycaemic effects of Pu-erh tea. Int. J. Food Sci. Technol. 54, 516-525 (2019).

8. Cabrera, C., Artacho, R. \& Gimenez, R. Beneficial effects of green tea-a review. J. Am. Coll. Nutr. 25, 79-99 (2006).

9. van de Laar, F. A. et al. a-Glucosidase Inhibitors for Patients With Type 2 Diabetes. Results a Cochrane Syst. Rev. Meta-Anal. 28, 154-163 (2005).

10. Deng, Y.-T., LinShiau, S.-Y., Shyur, L.-F. \& Lin, J.-K. Pu-erh tea polysaccharides decrease blood sugar by inhibition of alpha-glucosidase activity in vitro and in mice. Food Funct. 6, 1539-1546 (2015).

11. Li, D. Q., Qian, Z. M. \& Li, S. P. Inhibition of three selected beverage extracts on alpha-glucosidase and rapid identification of their active compounds using HPLC-DAD-MS/MS and biochemical detection. J. Agric. Food Chem. 58, 6608-6613 (2010)

12. Yang, X. H., Huang, J. A., Liu, Z. H., Mao, Q. L. \& Wu, W. L. Effect of Pu Erh tea on the activity of alpha glucosidase. Sci. Technol. Food Ind. 12, 122-124 (2012).

13. Huang, Q. et al. Studies on the bioactivity of aqueous extract of pu-erh tea and its fractions: in vitro antioxidant activity and alpha-glycosidase inhibitory property, and their effect on postprandial hyperglycemia in diabetic mice. Food Chem. Toxicol. 53, 75-83 (2013).

14. Wang, $X$. et al. Flavanols from the Camellia sinensis var. assamica and their hypoglycemic and hypolipidemic activities. Acta Pharm. Sin. B. 7, 342-346 (2017)

15. Zhang, D. Y., Huang, Y. W., Yuan, W. X. \& Zhou, H. J. Research of change of aamylase inhibitor content on Pu-erh tea pile-fermentation processing. Southwest China J. Agric. Sci. 21, 1282-1285 (2008).

16. Zhang, D. Y., Shao, W. F., Wang, Y. \& Zhou, H. J. Research on the Extraction Process of Pu-erh. Tea Fujian. 31, 20-22 (2009).

17. Zhang, D. Y., Shao, W. F., Liu, Z. H., Huang, Y. W. \& Shi, Z. P. Research on the chemical constituents of Pu-erh Tea and its inhibition effect on a-amylase. Southwest China J. Agric. Sci. 22, 52-54 (2009).

18. Zhou, L. J., Hou, C. Y., Qiao, Y. H. \& Zhao, J. Functional properties of Pu-erh tea with different storage years. Food Sci. 13, 19-22 (2010).

19. Liu, Y. H. \& Huang, H. H. The alpha amylase inhibition effect of Pu-erh tea and oolong tea extract. Food Ferment. Ind. 7, 54-57 (2010).

20. Wu, H., An, L. Y., Shi, C. \& Liu, C. Y. Study on a-amylase inhibitor in several teas. Jounral Southwest Natl Nat. Sci. Ed. 5, 768-770 (2011).

21. Standl, E. \& Schnell, O. Alpha-glucosidase inhibitors 2012 - cardiovascular considerations and trial evaluation. Diabetes Vasc. Dis. Res. 9, 163-169 (2012). 